Cite this: Nanoscale, 2013, 5, 9541

Received 11th June 2013

Accepted 27th July 2013

DOI: $10.1039 / \mathrm{c} 3 \mathrm{nr} 03014 \mathrm{~h}$

\section{Fast and robust infiltration of functional material inside titania nanotube layers: case study of a chalcogenide glass sensitizer $\dagger$}

\author{
Jan M. Macak, ${ }^{* a}$ Tomas Kohoutek, ${ }^{a}$ Lidong Wang $^{\mathrm{b}}$ and Radim Beranek ${ }^{\text {*b }}$
}

Fast and robust infiltration of anodic $\mathrm{TiO}_{2}$ nanotube layers with a model chalcogenide $\mathrm{As}_{3} \mathrm{~S}_{7}$ glass via spin-coating is reported for the first time. Effective sensitization leads to a significant visible light photocurrent response. This easy and cheap infiltration method can be extended for deposition of other absorbers into nanotubular layers.

Self-organized $\mathrm{TiO}_{2}$ nanotube layers, prepared by anodic oxidation of titanium in a suitable electrolyte, count among the most studied $\mathrm{TiO}_{2}$ materials in recent years. ${ }^{1}$ They have attracted huge scientific interest due to their intriguing properties, dimensional flexibility, low-cost reproducible production and scalability. In contrast to the early literature that reported only very low aspect ratio nanotubes, ${ }^{2,3}$ in past years careful engineering of the anodization conditions (mainly concerning the electrolyte) has facilitated the growth of high-aspect ratio nanotube layers within very short time scales, ${ }^{4-6}$ with $\mathrm{NH}_{4} \mathrm{~F}$ based ethylene glycol-water solution being the most popular and best performing electrolyte. Owing to their unique architecture, with a high degree of ordering, manifold applications of these nanotube layers have been demonstrated already in solar energy conversion, ${ }^{7-9}$ biomedicine,$^{10-12}$ photocatalysis ${ }^{13-15}$ and sensing. ${ }^{16}$ Notable examples also include various photoactive architectures in which $\mathrm{TiO}_{2}$ nanotube layers are used to collect electrons injected from chromophores, for example dyes ${ }^{17,18}$ and quantum dots based on $\mathrm{CdS},{ }^{19,20} \mathrm{CdSe}^{21-23}$ or carbon. ${ }^{24}$ Importantly, these sensitization approaches, particularly in the

\footnotetext{
${ }^{a}$ Department of General and Inorganic Chemistry, Faculty of Chemical Technology, University of Pardubice, Nam. Cs. Legii 565, 53002 Pardubice, Czech Republic. E-mail: jan.macak@upce.cz; Tel: +420466037401

${ }^{b}$ Faculty of Chemistry and Biochemistry, Ruhr University Bochum, Universitätstr. 150, D-44780 Bochum, Germany. E-mail: radim.beranek@rub.de; Fax: +49-234-3214174; Tel: $+49-234-3229431$

$\dagger$ Electronic supplementary information (ESI) available: Details on synthesis of $\mathrm{As}_{3} \mathrm{~S}_{7}$, photocurrent experiments; electronic absorption spectra and photocurrent measurements of $\mathrm{As}_{3} \mathrm{~S}_{7}$, potential dependence of photocurrents, EDX analysis. See DOI: 10.1039/c3nr03014h
}

case of non-molecular sensitizers, rely completely on the successful infiltration or filling of the nanotubes.

Nanotube infiltration protocols have so far focused mainly on chemical bath deposition, ${ }^{19-24}$ selective electrochemical reduction of the tubes ${ }^{25,26}$ or pulse deposition of monomers of conducting polymers inside the nanotubes with subsequent polymerization. ${ }^{27,28}$ However, these approaches are very limited in terms of choice of materials, and are only successful for tube layers with thicknesses of up to a few micrometres.

At present there is no robust infiltration or filling protocol known that is suitable for all nanotube generations, ${ }^{1}$ including tube layers with thicknesses up to several dozens of micrometres. One of the reasons for this is the very complex surface chemistry of $\mathrm{TiO}_{2}$ which is strongly related to its hydroxylation ${ }^{29}$ and which makes tube infiltration or filling rather unpredictable due to the surface reconstructions that occur during contact with water. ${ }^{30}$ Another obstacle to tube infiltration might be limited wetting of the tubes by solutions, particularly when freshly annealed. ${ }^{31}$ Finally, the presence of unremoved grassy layers $^{32}$ on the upper surface of the tubes (typical of nanotubes grown in ethylene glycol electrolytes) and small cracks may also cause some of the difficulties that are encountered when attempting to fill the tubes or coat their interior homogenously.

Therefore, in this paper we report for the first time, a very simple, yet very efficient and quantitative infiltration procedure for homogenous filling of nanotubes with a host material, which is based on spin-coating solutions with suitable rheological properties. This approach is versatile and allows for excellent control over the infiltration process. As a case study, we present the successful infiltration of $\mathrm{TiO}_{2}$ nanotubes with a chalcogenide glass $\mathrm{As}_{3} \mathrm{~S}_{7}$ which gives the tubes an efficient visible light photocurrent response. Thus this paper is also the first report of the effective sensitization of $\mathrm{TiO}_{2}$ nanotubes by an amorphous chalcogenide material.

The infiltration of our titania nanotube layers, employing the spin-coating of a chalcogenide glass solution, stems from the procedure used for the filling of nanostructured photonic crystal films described in the literature. ${ }^{33}$ The basic concept of 
infiltration of the chalcogenide material inside the titania nanotubes is shown in Fig. 1.

The amount of material that is infiltrated can be simply influenced by its concentration in the solution used for spincoating. As outlined in Fig. 1, the more concentrated the solution, the more material is deposited in the lower part of the tubes. Nevertheless, some material still remains on the tube walls where it forms a thin coating, thus yielding a high surface area sensitizer-nanotube interface. This is advantageous for more efficient light harvesting over the entire tube surface area. The assumption in Fig. 1 is corroborated by photoelectrochemical and EDX analysis as shown below.

Fig. 2a shows an example of the titania nanotube layer, that was used in the present study. All nanotube layers were prepared in the same fashion, by anodization of a Ti substrate for 24 hours in a pre-aged electrolyte containing $0.3 \mathrm{wt} \% \mathrm{NH}_{4} \mathrm{~F}$, ethylene glycol and $2 \mathrm{wt} \%$ water, using a two-electrode setup. Afterwards the samples were rinsed with isopropanol and sonicated for 5 minutes to remove the grassy layer on the surface. SEM investigations revealed no remnants of the grass after this procedure, all nanotube surfaces were clean and had open tube mouths. All nanotube layers were annealed, in a muffle oven at $450{ }^{\circ} \mathrm{C}$ for 3 hours, to obtain the anatase crystalline phase. The as-prepared nanotube layers were approximately $15 \mu \mathrm{m}$ thick and consisted of nanotubes with an average outer diameter of $125 \mathrm{~nm}$. In order to always achieve homogenous infiltration of the tubes by the chalcogenide, $1 \mathrm{ml}$ of the prepared $\mathrm{As}_{3} \mathrm{~S}_{7}$ solution was dropped onto a $1 \mathrm{~cm}^{2}$ area of the tubes which were attached tightly to the $\mathrm{Ti}$ substrate underneath. We used a range of concentrations of $\mathrm{As}_{3} \mathrm{~S}_{7}$ solutions in propylamine ${ }^{34,35}$ ranging from dilute solutions $(0.02 \mathrm{mg}$ $\left.\mathrm{ml}^{-1}\right)$ up to moderate concentrations $\left(0.166 \mathrm{mg} \mathrm{ml}^{-1}\right)$ with high viscosity. It was reported by Kohoutek et al. ${ }^{36}$ that chalcogenide glass mixtures with amine solvents form nanocolloidal solutions containing 2-8 $\mathrm{nm}$ glass clusters over a wide concentration range (comparable with that of our experiment). After spin-coating (2000 rpm) and subsequent vacuum drying at $125{ }^{\circ} \mathrm{C}$ for 10 hours, ${ }^{37,38}$ the as-prepared nanotube layers were subjected to photoelectrochemical measurements.

EDX analyses of the prepared samples confirmed that with increasing $\mathrm{As}_{3} \mathrm{~S}_{7}$ solution concentration, the nanotube layers became more enriched with $\mathrm{As}_{3} \mathrm{~S}_{7}$ inside the nanotubes. For
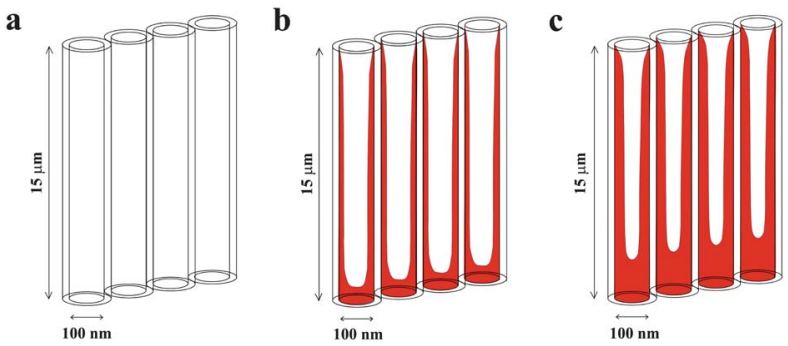

Fig. 1 The concept of infiltrating the titania nanotubes via a tailored spincoating procedure. (a) Plain tubes, (b) tubes with a low material content (corresponding to $0.1 \mathrm{~g} \mathrm{As}_{3} \mathrm{~S}_{7}$ per $\mathrm{ml}$ solution), (c) tubes with high material content (corresponding to $0.166 \mathrm{~g} \mathrm{As}_{3} \mathrm{~S}_{7}$ per $\mathrm{ml}$ solution).
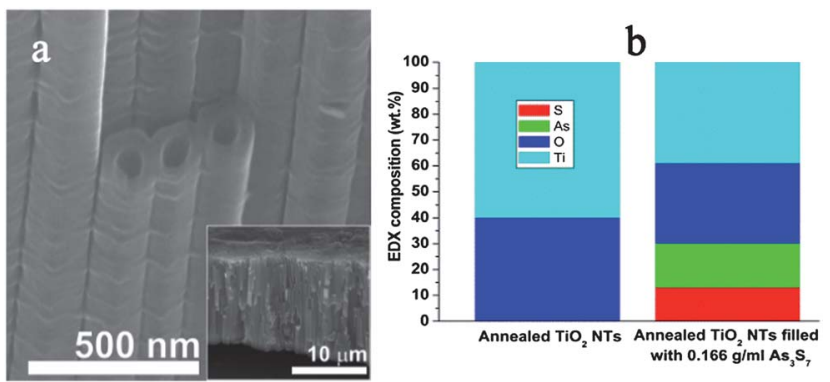

Fig. 2 (a) SEM image of crystalline $\mathrm{TiO}_{2}$ nanotubes infiltrated with amorphous $\mathrm{As}_{3} \mathrm{~S}_{7}$ glass, the inset shows the whole tube layer; (b) normalized compositional EDX profiles of $\mathrm{TiO}_{2}$ nanotube layers with and without infiltrated $\mathrm{As}_{3} \mathrm{~S}_{7}$ (mean values obtained from 20 measurements, standard deviation not exceeding $5 \%$ of the given values are displayed).

comparison, in Fig. 2b we show EDX data obtained from 20 measurements for a plain crystalline titania nanotube layer and the same tubes infiltrated with $\mathrm{As}_{3} \mathrm{~S}_{7}$ solution at the highest concentration, therefore, with the highest resulting $\mathrm{As}_{3} \mathrm{~S}_{7}$ content in the nanotubes. A line scan along a cracked piece of tube layer was used so that the values obtained represent the average elemental contents across the whole layer. It is noted that we could not obtain an SEM cross-sectional image of the internal cavity of the infiltrated tubes since the infiltrated tubes do not crack upon mechanical bending of the substrate or cutting with scissors in the way that they do when they are empty. Apparently, they have more mechanical stability due to the presence of the rigid amorphous glass material.

The bandgap energy of pristine spin-coated $\mathrm{As}_{3} \mathrm{~S}_{7}$ was determined as $2.45 \mathrm{eV}$ (506 $\mathrm{nm}$ ) using the Tauc formalism and assuming a non-direct optical transition (see ESI, Fig. S1 $\dagger$ ). In terms of electronic structure, the $\mathrm{As}_{3} \mathrm{~S}_{7}$ glass - similar to most chalcogenide glasses - is an intrinsic amorphous semiconductor with a low concentration of mobile carriers. ${ }^{39}$ Its Fermi level can be therefore expected to be positioned in the middle of the bandgap, which is corroborated by its photoelectrochemical behavior, showing a fingerprint typical of intrinsic semiconductors (see ESI, Fig. S2†). A film of pristine $\mathrm{As}_{3} \mathrm{~S}_{7}$ exhibits very low photocurrents $\left(<1 \mu \mathrm{A} \mathrm{cm}^{-2}\right)$ and their direction is switched during the potential scan from anodic to cathodic at approximately $+0.18 \mathrm{~V} v s$. NHE, coinciding with a Fermi level positioned in the middle of the bandgap.

In contrast, after coupling $\mathrm{As}_{3} \mathrm{~S}_{7}$ to $\mathrm{TiO}_{2}$ by infiltrating the titania nanotubes, a strong sensitizing effect is observed as shown in Fig. 3. The photocurrent response under UV light is enhanced by a factor of two as compared to pristine $\mathrm{TiO}_{2}$ nanotubes for all $\mathrm{As}_{3} \mathrm{~S}_{7}$ concentrations (Fig. 3a).

More importantly, significant photocurrent response can be observed in the visible light range, in particular for medium sensitizer concentrations ( 0.1 and $\left.0.125 \mathrm{~g} \mathrm{ml}^{-1}\right)$, where the highest IPCE (incident photon-to-current efficiency) values, up to $10 \%$, at around $470-480 \mathrm{~nm}$ are obtained (Fig. 3b). Given the bandgap of $\mathrm{As}_{3} \mathrm{~S}_{7}(2.45 \mathrm{eV} ; 506 \mathrm{~nm})$, it is interesting that the visible light-induced photocurrents are detectable even down to $650 \mathrm{~nm}(1.9 \mathrm{eV})$, this can be ascribed to the sub-bandgap Urbach tail absorption in $\mathrm{As}_{3} \mathrm{~S}_{7}$ (see ESI, Fig. S1 $\dagger$ ). The red shift of 

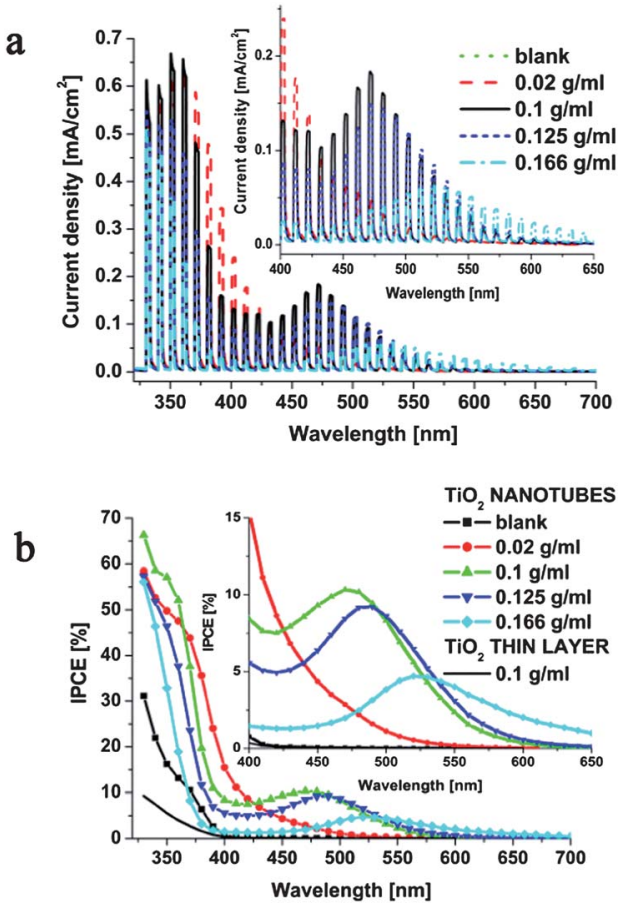

Fig. 3 (a) Photocurrent spectra and (b) incident photon-to-electron conversion efficiency recorded for nanotube layers infiltrated with different concentrations of $\mathrm{As}_{3} \mathrm{~S}_{7}$. The photocurrents were measured at $0.7 \mathrm{~V}$ vs. NHE in a $\mathrm{Na}_{2} \mathrm{SO}_{4}(0.1 \mathrm{M})$ electrolyte containing $\mathrm{KI}(0.1 \mathrm{M})$. For comparison, one typical IPCE curve acquired for a thin $\mathrm{TiO}_{2}$ layer sensitized with the $\mathrm{As}_{3} \mathrm{~S}_{7} 0.1 \mathrm{~g} \mathrm{ml}^{-1}$ solution is shown.

the sub-bandgap photocurrent response becomes more pronounced upon increasing the sensitizer content of the nanotubes. This red shift and the presence of maxima in the photo action spectra corroborate strongly our assumption that more material is deposited at the bottom of the tubes as the $\mathrm{As}_{3} \mathrm{~S}_{7}$ concentration used during spin-coating is increased (Fig. 1).

This can be explained in more detail as follows. Ultimately, the photocurrent generation efficiency at each wavelength will depend on the interplay between three factors: (i) light absorption, (ii) light penetration depth and (iii) the accessibility of electrolyte for the photogenerated holes. Obviously, the effect of the low-energy sub-bandgap absorption of $\mathrm{As}_{3} \mathrm{~S}_{7}$ (caused by its defect states) on the photocurrent response will be more pronounced with increased amounts of $\mathrm{As}_{3} \mathrm{~S}_{7}$ in the tubes. This will directly translate into a larger red shift of the photocurrent onset wavelength. On the other hand, since the photoelectrodes are irradiated from the front side (through the electrolyte) and since the light penetration depth increases with increasing wavelength, ${ }^{40}$ the recombination of photogenerated charges will be enhanced when these are either generated far from the underlying titanium substrate (long distance for electrons to travel), or when they are generated deep in the $\mathrm{As}_{3} \mathrm{~S}_{7}$ bulk at the bottom of the tubes (long pathway for the holes to travel to reach the electrolyte). Accordingly, depending on the interplay between the irradiation wavelength and the amount of $\mathrm{As}_{3} \mathrm{~S}_{7}$ at the bottom part of the tubes, a maximum in the photo action spectrum should be observed, with its position moving to longer wavelengths with increasing amounts of sensitizer, exactly as we observed (Fig. $3 \mathrm{~b}$ ). All in all, the medium sensitizer concentrations $\left(0.1\right.$ and $\left.0.125 \mathrm{~g} \mathrm{ml}^{-1}\right)$ clearly represent the optimum needed for achieving enhanced light absorption in the visible light range without compromising penetration of the nanotube layer by the electrolyte.

It should be noted that the presence of the aligned nanotubular architecture is absolutely crucial for achieving a reasonable sensitizing effect and photocurrent response. Accordingly, negligible photocurrent response in the visible region was observed when a thin crystalline compact $\mathrm{TiO}_{2}$ layer (prepared by annealing a Ti substrate in an oven at $450{ }^{\circ} \mathrm{C}$ for 3 hours) was spin-coated with $\mathrm{As}_{3} \mathrm{~S}_{7}$ (Fig. 3b). Clearly, here the very thin $\mathrm{As}_{3} \mathrm{~S}_{7}$ layer does not allow for sufficient light absorption, and only the nanostructured assembly is able to achieve efficient light harvesting in the visible light range.

The photocurrent generation under visible light irradiation of a $\mathrm{TiO}_{2}-\mathrm{As}_{3} \mathrm{~S}_{7}$ assembly can be summarized by the potential diagram in Fig. 4. Visible light-induced excitation of $\mathrm{As}_{3} \mathrm{~S}_{7}$ leads to injection of electrons into the conduction band of $\mathrm{TiO}_{2}$, whereby the holes can oxidize iodide in the solution $(E=1.3 \mathrm{~V}$ $v s$. NHE). ${ }^{41}$ The presence of iodide in the electrolyte was crucial to achieve long-term stability and avoid photo-corrosion of $\mathrm{As}_{3} \mathrm{~S}_{7}$, as confirmed by EDX measurements done before and after the photoelectrochemical measurement. It should be noted that this potential scheme is rather simplified. It cannot be ignored that the electron-hole pair in $\mathrm{As}_{3} \mathrm{~S}_{7}$ is more or less strongly bound (exciton), whereby the quasi-Fermi level of electrons is significantly less negative than the conduction band edge of $\mathrm{As}_{3} \mathrm{~S}_{7}$. Indeed, the photocurrent onset of the $\mathrm{TiO}_{2}-\mathrm{As}_{3} \mathrm{~S}_{7}$ photoelectrodes was determined to be at $c a$. $-0.4 \mathrm{~V} v$ s. NHE and $-0.18 \mathrm{~V} v s$. NHE at the wavelengths of $330 \mathrm{~nm}$ and $480 \mathrm{~nm}$, respectively (see ESI, Fig. S3†). Interestingly, under visible light irradiation $(480 \mathrm{~nm})$ small cathodic photocurrents were observed at negative potentials (the negative Fermi level of the Ti substrate), which suggests that under negative bias the electrons generated in $\mathrm{As}_{3} \mathrm{~S}_{7}$ can reduce oxygen in the solution, whereby the holes are "neutralized" by electrons injected from the Ti metal through $\mathrm{TiO}_{2}$, presumably via trap states under the conduction band edge. ${ }^{42}$

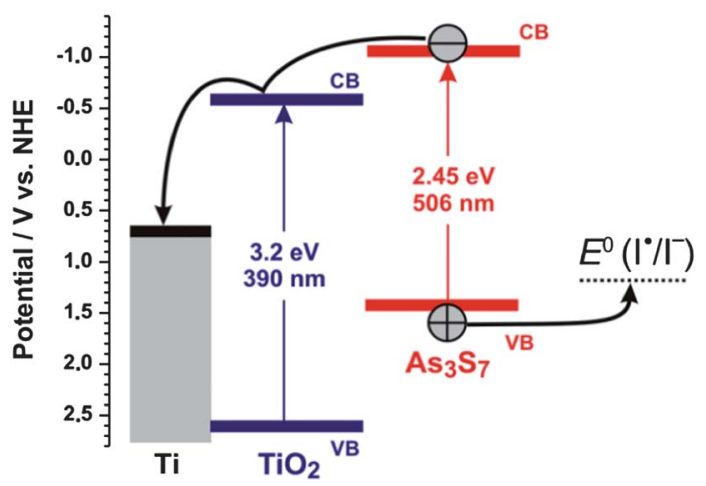

Fig. 4 Simplified potential diagram of the $\mathrm{TiO}_{2}-\mathrm{As}_{3} \mathrm{~S}_{7}$ interface at $\mathrm{pH} 7$ under an anodic bias of $0.7 \mathrm{~V}$ Vs. NHE. The position of the band edges of $\mathrm{As}_{3} \mathrm{~S}_{7}$ was determined by assuming that the position of the Fermi level was in the middle of the bandgap at $0.18 \mathrm{~V}$ vs. $\mathrm{NHE}$; the band edge positions of $\mathrm{TiO}_{2}$ were taken from the literature. ${ }^{43}$ 
It should be noted that, based on our experience, complete filling of the tubes by spin-coating is possible. For our model material, for instance, this can be achieved by using a highly concentrated chalcogenide solution with a high "honey-like" viscosity. This procedure, however, leads to formation of a solid lid on the tube array surface, and therefore has a detrimental effect on the photocurrent due to insufficient contact of the sensitizer with the electrolyte. Quantification of the $\mathrm{As}_{3} \mathrm{~S}_{7}$ content (or any other sensitizer) inside the nanotubes can be done by EDX, as shown in Fig. 1 and S4. $\dagger$ We will exploit the use of other sensitizers for tube filling in our future work and thoroughly analyze these systems by photoelectrochemistry, EDX and transmission electron microscopy.

\section{Conclusions}

We demonstrated that titania nanotube layers can be easily infiltrated with a chalcogenide glass sensitizer by spin-coating with its propylamine solution at an optimized concentration. This technique is low-cost and versatile, and allows the tube walls to be coated, or even for the tubes to be filled entirely, with better control than other approaches, such as electrodeposition, dipping or soaking. The prepared titania nanotubes sensitized with $\mathrm{As}_{3} \mathrm{~S}_{7}$ have shown significant photocurrent response under visible light irradiation. Taking into account the possibility of tuning the composition of the spin-coating chalcogenide glass solution and/or incorporating other (crystalline) colloidal sensitizers, we believe that the method presented here may pave the way for the fabrication of novel solar energy-converting architectures, photonic devices, $\mathrm{p}-\mathrm{n}$ junctions or drug-delivery assemblies based on $\mathrm{TiO}_{2}$ nanotubes.

\section{Acknowledgements}

J.M.M. and T.K. thank project CZ.1.07./2.3.00/20.0254 "ReAdMat - Research Team for Advanced Non-crystalline Materials", co-financed by the European Social Fund and state budget of the Czech Republic, for financial support. R.B. and L.W. acknowledge financial support from the MIWFT-NRW within the project "Anorganische Nanomaterialien für Anwendungen in der Photokatalyse”. The authors thank Dr Ronald Marschall for a fruitful discussion.

\section{Notes and references}

1 J. M. Macak, H. Tsuchiya, A. Ghicov, K. Yasuda, R. Hahn, S. Bauer and P. Schmuki, Curr. Opin. Solid State Mater. Sci., 2007, 1-2, 3-18.

2 V. Zwilling, M. Aucouturier and E. Darque-Ceretti, Electrochim. Acta, 1999, 35, 921-929.

3 R. Beranek, H. Hildebrand and P. Schmuki, Electrochem. Solid-State Lett., 2003, 6, B12-B14.

4 J. M. Macak, H. Tsuchiya, L. Taveira, S. Aldabergerova and P. Schmuki, Angew. Chem., Int. Ed., 2005, 44, 7463-7465.

5 S. P. Albu, A. Ghicov, J. M. Macak and P. Schmuki, Phys. Status Solidi RRL, 2007, 1, R65-R67.
6 S. So, K. Lee and P. Schmuki, J. Am. Chem. Soc., 2012, 134, 11316-11318.

7 J. M. Macak, H. Tsuchiya, A. Ghicov and P. Schmuki, Electrochem. Commun., 2005, 7, 1133-1137.

8 K. Zhu, N. R. Neale, A. Miedaner and A. J. Frank, Nano Lett., 2007, 7, 69-74.

9 S. So, K. Lee and P. Schmuki, Chem.-Eur. J., 2013, 19, 29662970.

10 J. M. Macak, H. Tsuchiya, L. Taveira, A. Ghicov and P. Schmuki, J. Biomed. Mater. Res., Part A, 2005, 75, 928-933.

11 J. Park, S. Bauer, K. A. Schlegel, F. W. Neukam, K. von der Mark and P. Schmuki, Small, 2009, 5, 666-671.

12 L. Zhao, L. Liu, Z. Wu, Y. Zhang and P. K. Chu, Biomaterials, 2012, 33, 2629-2641.

13 J. M. Macak, M. Zlamal, J. Krysa and P. Schmuki, Small, 2007, 3, 300-304.

14 N. Shreshta, J. M. Macak, F. Schmidt-Stein, R. Hahn, C. T. Mierke, B. Fabry and P. Schmuki, Angew. Chem., Int. Ed., 2009, 48, 969-972.

15 M. Altomare, M. Pozzi, M. Allieta, L. G. Bettini and E. Selli, Appl. Catal., B, 2013, 81-88, 136-137.

16 O. K. Varghese, D. Gong, M. Paulose, K. G. Ong and C. A. Grimes, Sens. Actuators, B, 2003, 93, 338-344.

17 B. O'Regan and M. Grätzel, Nature, 1991, 353, 737-739.

18 N. Robertson, Angew. Chem., Int. Ed., 2006, 45, 2338-2345.

19 W.-T. Sun, Y. Yu, H.-Y. Pan, X.-F. Gao, Q. Chen and L.-M. Pen, J. Am. Chem. Soc., 2008, 130, 1124-1125.

20 D. R. Baker and P. V. Kamat, Adv. Funct. Mater., 2009, 19, 805-811.

21 H. Zhang, X. Quan, S. Chen, H. Yu and N. Ma, Chem. Mater., 2009, 21, 3090-3095.

22 K. Shin, S. I. Seok, S. H. Im and J. H. Park, Chem. Commun., 2010, 46, 2385-2387.

23 Q. Shen, K. Katayama, T. Sawada, M. Yamaguchi and T. Toyoda, Jpn. J. Appl. Phys., 2006, 45, 5569-5574.

24 F. Wang, Y. Zhang, Y. Liu, Y. Wang, M. Shen, S.-T. Lee and Z. Kang, Nanoscale, 2013, 5, 1831-1835.

25 J. M. Macak, B. G. Gong, M. Hueppe and P. Schmuki, Adv. Mater., 2007, 19, 3027-3031.

26 J. M. Macak, C. Zollfrank, B. J. Rodriguez, H. Tsuchiya, M. Alexe, P. Greil and P. Schmuki, Adv. Mater., 2009, 21, 3121-3125.

27 D. Kowalski, A. Tighineanu and P. Schmuki, J. Mater. Chem., 2011, 21, 17909-17915.

28 D. Kowalski, S. P. Albu and P. Schmuki, RSC Adv., 2013, 3, 2154-2157.

29 U. Diebold, Surf. Sci. Rep., 2003, 48, 53-229.

30 D. Wang, L. Liu, F. Zhang, K. Tao, E. Pippel and K. Domen, Nano Lett., 2011, 11(9), 3649-3655.

31 D. Kim, J. M. Macak, F. Schmidt-Stein and P. Schmuki, Nanotechnology, 2008, 19, 305710.

32 D. Kim, A. Ghicov and P. Schmuki, Electrochem. Commun., 2008, 10(12), 1835-1838.

33 T. Kohoutek, J. Orava, T. Sawada and H. Fudouzi, J. Colloid Interface Sci., 2011, 353, 454-458.

34 G. C. Chern and I. Lauks, J. Appl. Phys., 1982, 53(10), 69796982. 
35 S. Song, J. Dua and C. B. Arnold, Opt. Express, 2010, 18(6), 5472-5480.

36 T. Kohoutek, T. Wagner, M. Frumar, A. Chrissanthopoulos, O. Kostadinova and S. N. Yannopoulos, J. Appl. Phys., 2008, 103(6), 063511-063516.

37 T. Kohoutek, T. Wagner, M. Vlcek, M. Vlcek and M. Frumar, J. Non-Cryst. Solids, 2006, 352, 1563-1566.

38 T. Kohoutek, T. Wagner, J. Orava, M. Krbal, T. Mates, A. Fejfar, S. O. Kasap and M. Frumar, J. Non-Cryst. Solids, 2007, 353, 1437-1440.
39 A. V. Kolobov, Photo-Induced Metastability in Amorphous Semiconductors, Wiley-VCH, Weinheim, 2003.

40 J. M. Macak, A. Ghicov, R. Hahn, T. Tsuchiya and P. Schmuki, J. Mater. Res., 2006, 21(11), 2824-2828.

41 P. Wardman, J. Phys. Chem. Ref. Data, 1989, 18, 1637-1755.

42 T. Berger, D. Monllor-Satoca, M. Jankulovska, T. LanaVillarreal and R. Gomez, ChemPhysChem, 2012, 13, 28242875.

43 R. Beranek, Adv. Phys. Chem., 2011, 786759, DOI: 10.1155/ 2011/7867591. 